\title{
Pressure Wave Propagation in Liquid-Filled Tubes of Viscoelastic Material
}

\author{
N. N. Kizilova
}

Received August 11, 2005

\begin{abstract}
The propagation of small-amplitude waves in a thick-walled long viscoelastic tube of variable cross-section, filled with a viscous incompressible fluid, is considered with account for wave reflection at the tube end in application to arterial pulse wave propagation. A solution is obtained in the form of expansions in a small parameter. The effect of the coefficient of wave reflection at the tube end and the wall material parameters on the fluid volume flow-rate and the tube wall displacement is investigated. It is shown that the volume flow-rate phase spectrum characteristics depend only slightly on the wall properties and can be used in clinical diagnostics for finding the reflection coefficient from pressure and flow-rate records.
\end{abstract}

Keywords: pulse wave, viscoelastic tube, wave reflection.

The propagation of pressure waves in liquid-filled compliant tubes has been intensely investigated in connection with the analysis of pulse waves in arteries [1-5]. The arterial vessels are multilayer tubes of viscoelastic orthotropic material and blood is a concentrated suspension of aggregating particles; therefore taking into account the fluid and wall rheology leads to fairly cumbersome mathematical models [6, 7] that can be investigated only numerically. However, many aspects associated with wave propagation and reflection in arterial beds can be studied using fairly simple models [8-12].

Blood flow in large arteries is characterized by high Reynolds numbers ( $R e \geq 500)$ and can be investigated on the basis of models of ideal incompressible fluid flow in compliant thin-walled tubes $(h / R \leq 0.1$, where $h$ is the wall thickness and $R$ is the inner radius of the tube). The viscoelastic properties of blood, associated with the presence of blood cells and high-molecular compounds, are manifested only in turbulent flow regimes [13]. When modeling flows in small arteries, the blood is mostly considered to be a homogeneous pseudoplastic fluid and the arteries to be thick-walled tubes of viscoelastic material $[1,6]$. Comparison of the results of simulating the pulse waves in arteries with account for the viscosity, compressibility and non-Newtonian characteristics of the blood and the compressibility and anisotropy of the wall material has shown that for the aorta and medium-sized arteries the main experimental laws of wave propagation and reflection can be satisfactorily described on the basis of the model of a thick-walled homogeneous isotropic viscoelastic tube filled with homogeneous incompressible viscous fluid [14].

The wave motion of the blood is generated by periodic heart contractions. Reflected waves arise in those segments in which the conductivities of the vessels are uncoordinated [1,6]. These are regions where the mechanical properties of the wall have changed due to pathologies (densifications, atherosclerotic plaques), as well as vessel bifurcations, contractions and expansions, normal or pathological (stenoses, aneurisms).

Thus, the intravascular pressure $p(t)$ curves recorded using micromanometers or obtained by measuring the artery diameter oscillations $d(t)$ by means of ultrasonic sensors are a result of the superposition of the incident wave and numerous reflected waves. The parameters of the $p(t)$ curves are determined by the reflection conditions; therefore, a detailed analysis and mechanical interpretation of the pressure waves is very important for clinical applications [15-18].

An important diagnostic parameter is the friction stress $\tau_{w}$ on the artery wall, whose value correlates with regions where atherosclerotic plaques are formed. The numerical calculations of $\tau_{w}$ values from the $d(t)$ 


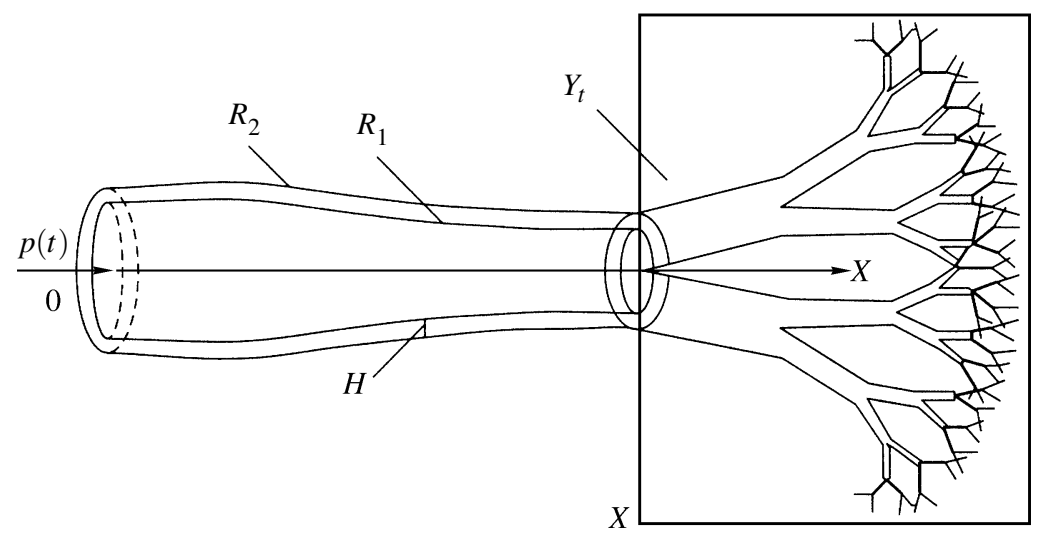

Fig. 1. Diagram of the arterial tree model as a tube with a terminal element $Y_{t}$

curves should be based on more detailed models that take into account the real structure and mechanical properties of the vascular wall material, presently lacking.

\section{FORMULATION OF THE PROBLEM}

We will consider an unsteady axisymmetric viscous incompressible fluid flow in an axisymmetric tube of variable cross-section similar in shape to a circular cylinder. We will assume that the tube wall consists of a homogeneous isotropic incompressible viscoelastic material. The tube length is $L$, the wall thickness $H$, and the inner radius in the unstressed state $R_{0}=R_{0}(x)$. We will assume that during wave transmission the tube cross-section remains circular with the inner and outer radii equal to $R_{1}$ and $R_{2}=R_{1}+H$, respectively. When applied to arteries, the dependence $R_{0}(x)$ may take into account their natural gradual narrowing, which is especially important for the aorta and large arteries. The outer surface of the tube is assumed to be attached to the surrounding tissues.

At the end $x=L$ the tube is sequentially connected with a tube of different radius or a bifurcation of tubes that continue the arterial bed treated as a terminal element with a given complex characteristic admittance $Y_{t}$ (Fig. 1). The equations of fluid motion are

$$
\begin{aligned}
& \operatorname{div}(\mathbf{v})=0 \\
& \frac{\partial \mathbf{v}}{\partial t}+(\mathbf{v} \boldsymbol{\nabla}) \mathbf{v}=-\frac{1}{\rho_{f}} \boldsymbol{\nabla} p+v \Delta \mathbf{v}
\end{aligned}
$$

where $\mathbf{v}=\left(v_{r}, 0, v_{x}\right), p, \rho_{f}$, and $v$ are the velocity, hydrostatic pressure, density and kinematic viscosity of the fluid.

The equation of tube wall motion is

$$
\rho_{s} \frac{\partial^{2} \mathbf{u}}{\partial t^{2}}=\operatorname{div} \boldsymbol{\Sigma}
$$

where $\mathbf{u}=\left(u_{r}, 0, u_{x}\right)$ is the displacement vector, $\rho_{s}$ is the material density, and $\boldsymbol{\Sigma}$ is the wall stress tensor.

We will describe the viscoelastic properties of the wall material by means of a three-element rheological model which takes account of stress and strain relaxation [13]:

$$
\lambda_{1} \frac{\delta \boldsymbol{\sigma}}{\delta t}+\boldsymbol{\sigma}=2 G\left(\mathbf{e}+\lambda_{2} \frac{\delta \mathbf{e}}{\delta t}\right), \quad \boldsymbol{\sigma}=\boldsymbol{\Sigma}+p_{s} \mathbf{I}
$$

where $p_{s}$ is the hydrostatic pressure in the wall, $\mathbf{I}$ is the unit tensor, $\lambda_{1,2}$ are the relaxation and retardation times, $G$ is the shear modulus, $\mathbf{e}$ is the wall strain tensor, and by $\delta / \delta t$ we denote the differentiation of a tensor with respect to time. We will consider the constitutive equation (1.4) in the linear approximation and in what follows, instead of $\delta / \delta t$, use the partial time derivative. 
From (1.3), (1.4) we obtain the system of equations of wall motion in displacements, which in the cylindrical coordinate system tied to the tube takes the form [19]:

$$
\begin{gathered}
\left(1+\lambda_{1} \frac{\partial}{\partial t}\right)\left(\rho_{s} \frac{\partial^{2} u_{r}}{\partial t^{2}}+\frac{\partial p_{s}}{\partial r}\right)=G\left(1+\lambda_{2} \frac{\partial}{\partial t}\right)\left(\frac{\partial^{2} u_{r}}{\partial r^{2}}+\frac{1}{r} \frac{\partial u_{r}}{\partial r}-\frac{u_{r}}{r^{2}}+\frac{\partial^{2} u_{r}}{\partial x^{2}}\right) \\
\left(1+\lambda_{1} \frac{\partial}{\partial t}\right)\left(\rho_{s} \frac{\partial^{2} u_{x}}{\partial t^{2}}+\frac{\partial p_{s}}{\partial x}\right)=G\left(1+\lambda_{2} \frac{\partial}{\partial t}\right)\left(\frac{\partial^{2} u_{x}}{\partial r^{2}}+\frac{1}{r} \frac{\partial u_{x}}{\partial r}+\frac{\partial^{2} u_{x}}{\partial x^{2}}\right)
\end{gathered}
$$

We close the model with the material incompressibility condition

$$
\frac{1}{r} \frac{\partial}{\partial r}\left(r u_{r}\right)+\frac{\partial u_{x}}{\partial x}=0
$$

As boundary conditions for system (1.1), (1.2), (1.5)-(1.7) we will use the conditions of axial symmetry of the profile and velocity boundedness on the tube axis

$$
r=0: \quad v_{r}=0, \quad\left|v_{x}\right|<\infty
$$

the conditions of velocity and stress continuity on the fluid-wall interface

$$
\begin{aligned}
& r=R_{1}: \quad v_{r}=\frac{\partial u_{r}}{\partial t}, \quad v_{x}=\frac{\partial u_{x}}{\partial t} \\
& \left(1+\lambda_{1} \frac{\partial}{\partial t}\right)\left(p_{s}-p+2 v \rho_{f} \frac{\partial v_{r}}{\partial r}\right)=2 G\left(1+\lambda_{2} \frac{\partial}{\partial t}\right) \frac{\partial u_{r}}{\partial r} \\
& v \rho_{f}\left(1+\lambda_{1} \frac{\partial}{\partial t}\right)\left(\frac{\partial v_{r}}{\partial x}+\frac{\partial v_{x}}{\partial r}\right)=G\left(1+\lambda_{2} \frac{\partial}{\partial t}\right)\left(\frac{\partial u_{r}}{\partial x}+\frac{\partial u_{x}}{\partial r}\right)
\end{aligned}
$$

and the condition of tube wall attachment to the surrounding non-deformable medium

$$
r=R_{2}: u_{r, x}=0
$$

In relations (1.9) we neglect the difference in direction between the vector tangent to the fluid-wall interface and the longitudinal axis of the tube, which is related with the assumption that the tube is almost cylindrical in shape.

At the tube inlet a pressure wave is given:

$$
x=0: \frac{2}{R_{1}^{2}} \int_{0}^{R_{1}} r p(t, r, 0) d r=p_{0}(t)
$$

From the definition of the inlet characteristic admittance $Y_{t}=Q /\langle P\rangle$ of a terminal element, where $Q$ is the volume flow-rate and $\langle P\rangle$ is the pressure averaged over the cross-section, there follows the tube end condition

$$
x=L: \quad Y_{t} \int_{0}^{R_{1}} r p(t, r, x) d r=\pi R_{1}^{2} \int_{0}^{R_{1}} r v_{x}(t, r, x) d r
$$

The function $p_{0}(t)$ can be specified explicitly on the basis of the experimentally measured pressure oscillations in various cross-sections of human and animal arteries [1]. In theoretical models the expression for $p_{0}(t)$ is usually given in the form of a Fourier expansion. Since the pulse curves can be satisfactorily described by four to six expansion harmonics, this method of inlet condition assignment makes it possible 
to calculate real blood flow velocity profiles and compare them with the measurement results $[1,6]$. Previously, the problem (1.1) (1.2), (1.5)-(1.12) was investigated for the Voigt model $\left(\lambda_{1}=0\right)$ and the linearized equations (1.2) [8] and in the nonlinear formulation for an inviscid fluid and $\lambda_{1,2}=0[10,11]$.

For human arteries, the characteristic tube dimensions, wall displacements, and blood flow velocities are equal $[1,6]$ to

$$
\begin{aligned}
& r_{0}=10^{-4}-10^{-2} \mathrm{~m}, \quad H^{*} / r_{0}=10^{-3}-5 \cdot 10^{-1}, \quad r_{0} / L=2 \cdot 10^{-2}-2 \cdot 10^{-1} \\
& v_{x}^{*}=2 \cdot 10^{-3}-6 \cdot 10^{-1} \mathrm{~m} / \mathrm{s}, \quad u_{r}^{*} / r_{0}=(1-8) \cdot 10^{-2}, \quad r_{0}=\frac{1}{L} \int_{0}^{L} R_{0}(x) d x
\end{aligned}
$$

where the asterisks denote the characteristic values of the corresponding quantities and $r_{0}$ is the inner radius in the unstressed state averaged over the tube length. Thus, we may assume that the tube is long, that is, $r_{0} / L \equiv \varepsilon \ll 1$, and the radial displacements of the wall are small and of the same order $u_{r}^{*} / r_{0}=\varepsilon$.

We will transform boundary conditions (1.10), taking into account that the wall displacements are uniquely determined by the interaction of the fluid with the wall and the mechanical properties of the wall material. At an arbitrary moment the inner radius of the tube $R_{1}(t, x)=R_{0}(x)+u_{r}\left(t, R_{0}(x), x\right)$. Expanding in (1.9) the functions of the form $f\left(t, R_{1}(t, x), x\right)$, where $f=\left\{u_{r}, u_{x}, v_{r}, v_{x}, p, p_{s}\right\}$, in Taylor series and neglecting terms of the order of $u_{r}^{2}$, we replace (1.9) by the relations

$$
\begin{gathered}
r=R_{0}(x): v_{r}+\frac{\partial v_{r}}{\partial r} u_{r}=\frac{\partial u_{r}}{\partial t}+\frac{\partial u_{r}}{\partial r} \frac{\partial u_{r}}{\partial t}+\frac{\partial^{2} u_{r}}{\partial t \partial r} u_{r} \\
v_{x}+\frac{\partial v_{x}}{\partial r} u_{r}=\frac{\partial u_{x}}{\partial t}+\frac{\partial u_{x}}{\partial r} \frac{\partial u_{r}}{\partial t}+\frac{\partial^{2} u_{x}}{\partial t \partial r} u_{r} \\
\left(1+\lambda_{1} \frac{\partial}{\partial t}\right)\left(p_{s}-p+u_{r} \frac{\partial}{\partial r}\left(p_{s}-p\right)+2 v \rho_{f}\left(\frac{\partial v_{r}}{\partial r}+u_{r} \frac{\partial^{2} v_{r}}{\partial r^{2}}+\frac{\partial v_{r}}{\partial r} \frac{\partial u_{r}}{\partial r}\right)\right)= \\
2 G\left(1+\lambda_{2} \frac{\partial}{\partial t}\right)\left(\frac{\partial u_{r}}{\partial r}+u_{r} \frac{\partial^{2} u_{r}}{\partial r^{2}}+\left(\frac{\partial u_{r}}{\partial r}\right)^{2}\right) \\
v \rho_{f}\left(1+\lambda_{1} \frac{\partial}{\partial t}\right)\left(\frac{\partial v_{r}}{\partial x}+\frac{\partial v_{x}}{\partial r}+u_{r} \frac{\partial^{2} v_{r}}{\partial r \partial x}+\frac{\partial v_{r}}{\partial r} \frac{\partial u_{r}}{\partial x}+u_{r} \frac{\partial^{2} v_{x}}{\partial r^{2}}+\frac{\partial v_{x}}{\partial r} \frac{\partial u_{r}}{\partial r}\right)= \\
G\left(1+\lambda_{2} \frac{\partial}{\partial t}\right)\left(\frac{\partial u_{r}}{\partial x}+\frac{\partial u_{x}}{\partial r}+u_{r} \frac{\partial^{2} u_{r}}{\partial r \partial x}+\frac{\partial u_{r}}{\partial r} \frac{\partial u_{r}}{\partial x}+u_{r} \frac{\partial^{2} u_{x}}{\partial r^{2}}+\frac{\partial u_{x}}{\partial r} \frac{\partial u_{r}}{\partial r}\right)
\end{gathered}
$$

We will solve the problem in the dimensionless variables

$$
x^{\circ}=\frac{x}{L}, \quad r^{\circ}=\frac{r}{r_{0}}, \quad t^{\circ}=\frac{t c}{L}, \quad u_{r, x}^{\circ}=\frac{u_{r, x}}{u_{r, x}^{*}}, \quad v_{r, x}^{\circ}=\frac{v_{r, x}}{v_{r, x}^{*}}, \quad p^{\circ}=\frac{p}{p^{*}}, \quad p_{s}^{\circ}=\frac{p}{p_{s}^{*}}
$$

where $c$ is the pulse wave propagation velocity. Since the numerical estimates yield $u_{r}^{*}=\varepsilon r_{0}$, from Eq. (1.7) it follows that $u_{x}^{*}=u_{r}^{*} / \varepsilon=r_{0}$. From the first two relations (1.9) we find that $v_{r}^{*}=c \varepsilon^{2}$ and $v_{x}^{*}=c \varepsilon$. We will assume that $p^{*}, p_{s}^{*}=\rho_{f} c^{2} \varepsilon$ and rewrite (1.2), (1.5), (1.6) in dimensionless form (in what follows we will omit the superscripts that denote the dimensionless variables):

$$
\begin{gathered}
\varepsilon^{2} \frac{\partial v_{r}}{\partial t}+\varepsilon^{3} v_{r} \frac{\partial v_{r}}{\partial r}+\varepsilon^{3} v_{x} \frac{\partial v_{r}}{\partial x}=-\frac{\partial p}{\partial r}+\frac{\varepsilon^{2}}{\operatorname{Re}}\left(\frac{\partial^{2} v_{r}}{\partial r^{2}}+\frac{1}{r} \frac{\partial v_{r}}{\partial r}-\frac{v_{r}}{r^{2}}+\varepsilon^{2} \frac{\partial^{2} v_{r}}{\partial x^{2}}\right) \\
\frac{\partial v_{x}}{\partial t}+\varepsilon v_{r} \frac{\partial v_{x}}{\partial r}+\varepsilon v_{x} \frac{\partial v_{x}}{\partial x}=-\frac{\partial p}{\partial x}+\frac{1}{\operatorname{Re}}\left(\frac{\partial^{2} v_{x}}{\partial r^{2}}+\frac{1}{r} \frac{\partial v_{x}}{\partial r}+\varepsilon^{2} \frac{\partial^{2} v_{x}}{\partial x^{2}}\right)
\end{gathered}
$$




$$
\begin{gathered}
\left(1+\alpha_{1} \frac{\partial}{\partial t}\right)\left(\varepsilon^{2} \rho \frac{\partial^{2} u_{r}}{\partial t^{2}}+\frac{\partial p_{s}}{\partial r}\right)=\frac{\Gamma \varepsilon^{2}}{\operatorname{Re}}\left(1+\alpha_{2} \frac{\partial}{\partial t}\right)\left(\frac{\partial^{2} u_{r}}{\partial r^{2}}+\frac{1}{r} \frac{\partial u_{r}}{\partial r}-\frac{u_{r}}{r^{2}}+\varepsilon^{2} \frac{\partial^{2} u_{r}}{\partial x^{2}}\right) \\
\left(1+\alpha_{1} \frac{\partial}{\partial t}\right)\left(\rho \frac{\partial^{2} u_{x}}{\partial t^{2}}+\frac{\partial p_{s}}{\partial x}\right)=\frac{\Gamma}{\operatorname{Re}}\left(1+\alpha_{2} \frac{\partial}{\partial t}\right)\left(\frac{\partial^{2} u_{x}}{\partial r^{2}}+\frac{1}{r} \frac{\partial u_{x}}{\partial r}+\varepsilon^{2} \frac{\partial^{2} u_{x}}{\partial x^{2}}\right) \\
\operatorname{Re}=\frac{c \varepsilon R}{v}, \quad \Gamma=\frac{G r_{0}}{\mu c}, \quad \alpha_{i}=\frac{\lambda_{i} c}{L}, \quad \rho=\frac{\rho_{s}}{\rho_{f}}
\end{gathered}
$$

where $\operatorname{Re}$ is the Reynolds number, $\Gamma$ is the dimensionless shear modulus, $\mu$ is the fluid viscosity, and $i=1$, 2.

We will rewrite conditions (1.8), (1.10)-(1.14) in dimensionless form. Relations (1.1), (1.7), (1.8), and (1.10) then remain unchanged, whereas conditions (1.13) and (1.14) take the form:

$$
\begin{gathered}
r=a: \quad v_{r}+\varepsilon u_{r} \frac{\partial v_{r}}{\partial r}=\frac{\partial u_{r}}{\partial t}+\varepsilon v_{r} \frac{\partial u_{r}}{\partial r}, \quad v_{x}+\varepsilon u_{r} \frac{\partial v_{x}}{\partial r}=\frac{\partial u_{x}}{\partial t}+\varepsilon v_{r} \frac{\partial u_{x}}{\partial r} \\
\left(1+\alpha_{1} \frac{\partial}{\partial t}\right)\left(p_{s}-p+\varepsilon u_{r} \frac{\partial}{\partial r}\left(p_{s}-p\right)+\frac{2 \varepsilon^{2}}{\operatorname{Re}}\left(\frac{\partial v_{r}}{\partial r}+\varepsilon u_{r} \frac{\partial^{2} v_{r}}{\partial r^{2}}+\varepsilon \frac{\partial v_{r}}{\partial r} \frac{\partial u_{r}}{\partial r}\right)\right)= \\
\frac{2 \Gamma \varepsilon}{\operatorname{Re}}\left(1+\alpha_{2} \frac{\partial}{\partial t}\right)\left(\frac{\partial u_{r}}{\partial r}+\varepsilon u_{r} \frac{\partial^{2} u_{r}}{\partial r^{2}}+\varepsilon\left(\frac{\partial u_{r}}{\partial r}\right)^{2}\right) \\
\varepsilon\left(1+\alpha_{1} \frac{\partial}{\partial t}\right)\left(\varepsilon^{2} \frac{\partial v_{r}}{\partial x}+\frac{\partial v_{x}}{\partial r}+\varepsilon^{3}\left(u_{r} \frac{\partial^{2} v_{r}}{\partial r \partial x}+\frac{\partial v_{r}}{\partial r} \frac{\partial u_{r}}{\partial x}\right)+\varepsilon\left(u_{r} \frac{\partial^{2} v_{x}}{\partial r^{2}}+\frac{\partial v_{x}}{\partial r} \frac{\partial u_{r}}{\partial r}\right)\right)= \\
\Gamma\left(1+\alpha_{2} \frac{\partial}{\partial t}\right)\left(\varepsilon^{2} \frac{\partial u_{r}}{\partial x}+\frac{\partial u_{x}}{\partial r}+\varepsilon^{3}\left(u_{r} \frac{\partial^{2} u_{r}}{\partial r \partial x}+\frac{\partial u_{r}}{\partial r} \frac{\partial u_{r}}{\partial x}\right)+\varepsilon\left(u_{r} \frac{\partial^{2} u_{x}}{\partial r^{2}}+\frac{\partial u_{x}}{\partial r} \frac{\partial u_{r}}{\partial r}\right)\right) \\
r_{i}=\frac{R_{i}}{r_{0}}, \quad a(x)=\frac{R_{0}(x)}{r_{0}}, \quad i=1,2
\end{gathered}
$$

Conditions (1.11) and (1.12) take the dimensionless form

$$
\begin{aligned}
& x=0: \quad 2 \int_{0}^{a_{0}} r p(t, r, 0) d r+\left.\left.2 \varepsilon a_{0} p\right|_{r=a_{0}} u_{r}\right|_{r=a_{0}}=\left(a_{0}^{2}+\left.2 \varepsilon a_{0} u_{r}\right|_{r=a_{0}}\right) p_{0}(t), \quad a_{0}=a(0) \\
& x=1: \quad y_{t}\left(\int_{0}^{a_{1}} r p(t, r, 1) d r+\left.\left.\varepsilon a_{1} p\right|_{r=a_{1}} u_{r}\right|_{r=a_{1}}\right)=\left(a_{1}^{2}+\left.2 \varepsilon a_{1} u_{r}\right|_{r=a_{1}}\right) \times \\
& \left(\int_{0}^{a_{1}} r v_{x}(t, r, 1) d r+\left.\left.\varepsilon a_{1} v_{x}\right|_{r=a} u_{r}\right|_{r=a_{1}}\right), \quad a_{1}=a(1)
\end{aligned}
$$

where $y_{t}=Y_{t} / Y_{t}^{*}$ is the dimensionless admittance of the terminal element and $Y^{*}=\pi r_{0}^{2} /\left(\rho_{f} c\right)$ is the characteristic conductivity of the tube. 


\section{METHOD OF SOLUTION}

We will seek the problem solution in the form of expansions in the small parameter $\varepsilon$

$$
g=g^{(0)}+\varepsilon g^{(1)}+\varepsilon^{2} g^{(2)}+\ldots
$$

Substituting expressions of form (2.1) in relations (1.1), (1.7), (1.8), (1.15)-(1.21) and equating to zero the multipliers of like powers of the small parameter, we obtain systems of equations for the zeroth and first approximations of the problem and the corresponding boundary conditions. For the zeroth approximation

$$
\begin{aligned}
& \frac{\partial v_{r}^{(0)}}{\partial r}+\frac{v_{r}^{(0)}}{r}+\frac{\partial v_{x}^{(0)}}{\partial x}=0, \quad \frac{\partial u_{r}^{(0)}}{\partial r}+\frac{u_{r}^{(0)}}{r}+\frac{\partial u_{x}^{(0)}}{\partial x}=0 \\
& \frac{\partial p^{(0)}}{\partial r}=0, \quad \frac{\partial v_{x}^{(0)}}{\partial t}+\frac{\partial p^{(0)}}{\partial x}-\frac{1}{\operatorname{Re}}\left(\frac{\partial^{2} v_{x}^{(0)}}{\partial r^{2}}+\frac{1}{r} \frac{\partial v_{x}^{(0)}}{\partial r}\right)=0 \\
& \frac{\partial p_{s}^{(0)}}{\partial r}+\alpha_{1} \frac{\partial^{2} p_{s}^{(0)}}{\partial r \partial t}=0 \\
& \left(1+\alpha_{1} \frac{\partial}{\partial t}\right)\left(\rho \frac{\partial^{2} u_{x}^{(0)}}{\partial t^{2}}+\frac{\partial p_{s}^{(0)}}{\partial x}\right)=\frac{\Gamma}{\operatorname{Re}}\left(1+\alpha_{2} \frac{\partial}{\partial t}\right)\left(\frac{\partial^{2} u_{x}^{(0)}}{\partial r^{2}}+\frac{1}{r} \frac{\partial u_{x}^{(0)}}{\partial r}\right) \\
& r=0: v_{r}^{(0)}=0, \quad\left|v_{x}^{(0)}\right|<\infty \\
& r=a(x): \quad v_{r}^{(0)}=\frac{\partial u_{r}^{(0)}}{\partial t}, \quad v_{x}^{(0)}=\frac{\partial u_{x}^{(0)}}{\partial t} \quad\left(1+\alpha_{2} \frac{\partial}{\partial t}\right) \frac{\partial u_{x}}{\partial r}=0 \\
& \left(1+\alpha_{1} \frac{\partial}{\partial t}\right)\left(p_{s}^{(0)}-p^{(0)}\right)=0, \quad\left(u_{r, x}^{a}=0\right. \\
& r=r_{2}: \quad \int_{0}^{(0)} r p^{(0)} d r=a^{2} \int_{0}^{a} r v_{x}^{(0)} d r \\
& x=0: 2 p_{0}^{(0)} d r=a^{2} p_{0}, \quad x=1: y_{t}
\end{aligned}
$$

For the first approximation

$$
\begin{aligned}
& \frac{\partial v_{r}^{(1)}}{\partial r}+\frac{v_{r}^{(1)}}{r}+\frac{\partial v_{x}^{(1)}}{\partial x}=0, \quad \frac{\partial u_{r}^{(1)}}{\partial r}+\frac{u_{r}^{(1)}}{r}+\frac{\partial u_{x}^{(1)}}{\partial x}=0 \\
& \frac{\partial v_{x}^{(1)}}{\partial t}+\frac{\partial p^{(1)}}{\partial x}-\frac{1}{\operatorname{Re}}\left(\frac{\partial^{2} v_{x}^{(1)}}{\partial r^{2}}+\frac{1}{r} \frac{\partial v_{x}^{(1)}}{\partial r}\right)=-v_{x}^{(0)} \frac{\partial v_{x}^{(0)}}{\partial x}-v_{r}^{(0)} \frac{\partial v_{x}^{(0)}}{\partial r} \\
& \frac{\partial p^{(1)}}{\partial r}=0 \\
& \left(1+\alpha_{1} \frac{\partial}{\partial t}\right)\left(\rho \frac{\partial^{2} u_{x}^{(1)}}{\partial t^{2}}+\frac{\partial p_{s}^{(1)}}{\partial x}\right)=\frac{\Gamma}{\operatorname{Re}}\left(1+\alpha_{2} \frac{\partial}{\partial t}\right)\left(\frac{\partial^{2} u_{x}^{(1)}}{\partial r^{2}}+\frac{1}{r} \frac{\partial u_{x}^{(1)}}{\partial r}\right) \\
& \left(1+\alpha_{1} \frac{\partial}{\partial t}\right) \frac{\partial p_{s}^{(1)}}{\partial r}=\frac{\Gamma}{\operatorname{Re}}\left(1+\alpha_{2} \frac{\partial}{\partial t}\right)\left(\frac{\partial^{2} u_{r}^{(0)}}{\partial r^{2}}+\frac{1}{r} \frac{\partial u_{r}^{(0)}}{\partial r}-\frac{u_{r}^{(0)}}{r^{2}}\right) \\
& r=0: v_{r}^{(1)}=0, \quad\left|v_{x}^{(1)}\right|<\infty
\end{aligned}
$$




$$
\begin{aligned}
& r=a(x): v_{r}^{(1)}-\frac{\partial u_{r}^{(1)}}{\partial t}=\frac{\partial u_{r}^{(0)}}{\partial t} \frac{\partial u_{r}^{(0)}}{\partial r}-u_{r}^{(0)} \frac{\partial v_{r}^{(0)}}{\partial r}+u_{r}^{(0)} \frac{\partial^{2} u_{r}^{(0)}}{\partial t \partial r} \\
& v_{x}^{(1)}-\frac{\partial u_{x}^{(1)}}{\partial t}=\frac{\partial u_{r}^{(0)}}{\partial t} \frac{\partial u_{x}^{(0)}}{\partial r}-u_{r}^{(0)} \frac{\partial v_{x}^{(0)}}{\partial r}+u_{r}^{(0)} \frac{\partial^{2} u_{x}^{(0)}}{\partial t \partial r} \\
& \left(1+\alpha_{1} \frac{\partial}{\partial t}\right)\left(p_{s}^{(1)}-p^{(1)}\right)=\frac{2 \Gamma}{\operatorname{Re}}\left(1+\alpha_{2} \frac{\partial}{\partial t}\right) \frac{\partial u_{r}^{(0)}}{\partial r}- \\
& \left(1+\alpha_{1} \frac{\partial}{\partial t}\right)\left(u_{r}^{(0)} \frac{\partial}{\partial r}\left(p_{s}^{(0)}-p^{(0)}\right)\right) \\
& \Gamma\left(1+\alpha_{2} \frac{\partial}{\partial t}\right) \frac{\partial u_{x}^{(1)}}{\partial r}=\left(1+\alpha_{1} \frac{\partial}{\partial t}\right) \frac{\partial v_{x}^{(0)}}{\partial r} \\
& r=r_{2}: u_{r, x}^{(1)}=0 \\
& x=0: 2 \int_{0}^{a} r p^{(1)} d r+\left.\left.2 a u_{r}^{(0)}\right|_{r=a} p^{(0)}\right|_{r=a}=\left.2 a u_{r}^{(0)}\right|_{r=a} p_{0} \\
& x=1: y_{t}\left(\int_{0}^{a} r p^{(1)} d r+\left.\left.a p^{(0)}\right|_{r=a} u_{r}^{(0)}\right|_{r=a}\right)=\left.2 a u_{r}^{(0)}\right|_{r=a} \int_{0}^{a} r v_{x}^{(0)} d r+ \\
& a^{2} \int_{0}^{a} r v_{x}^{(1)} d r+\left.\left.a^{3} v_{x}^{(0)}\right|_{r=a} u_{r}^{(0)}\right|_{r=a}
\end{aligned}
$$

\section{SOLUTION OF THE PROBLEM FOR THE ZEROTH AND FIRST APPROXIMATIONS}

The zeroth-approximation problem (2.2)-(2.10) generalizes the classical problem [8] to include the case of a viscoelastic wall material and a tube of finite length. At the tube inlet a pressure wave $p_{0}(t)=p_{0} e^{i \omega t}$ is given and the solution can be found in the form:

$$
g(t, r, x)=g^{*}(r, x) e^{i \omega t}, \quad g=\left\{u_{r}^{(0)}, u_{x}^{(0)}, v_{r}^{(0)}, v_{x}^{(0)}, p^{(0)}, p_{s}^{(0)}\right\}
$$

From the first relation (2.3) and (2.4) it follows that $p^{*}=p^{*}(x)$ and $p_{s}^{*}=p_{s}^{*}(x)$. Problem (2.2)-(2.10) can be separated and the solution of the second equation (2.3) which satisfies the second condition (2.6) has the form:

$$
v_{x}^{*}(r, x)=C_{1}(x) J_{0}(\gamma r)+\frac{i}{\omega}\left(p^{*}\right)^{\prime}, \quad \gamma=(-i \omega \mathrm{Re})^{0.5}
$$

where $J_{n}$ is a Bessel function of the first kind and $C_{1}(x)$ is an unknown function. Substituting (3.1) in the first relation (2.2), we obtain an equation for $v_{r}^{*}(r, x)$ whose solution with account for the first condition (2.6) has the form:

$$
v_{r}^{*}(r, x)=-\frac{J_{1}(\gamma r)}{\gamma} C_{1}^{\prime}(x)-\frac{i r}{2 \omega}\left(p^{*}\right)^{\prime \prime}
$$

The solution of Eq. (2.5) which satisfies (2.9) is

$$
\begin{aligned}
& u_{x}^{*}(r, x)=C_{3}(x) Y_{0}(\beta r)+C_{4}(x) J_{0}(\beta r)+\frac{1}{\rho \omega^{2}}\left(p_{s}^{*}\right)^{\prime} \\
& C_{4}(x)=-J_{0}^{-1}\left(\beta r_{2}\right)\left(\frac{1}{\rho \omega^{2}}\left(p_{s}^{*}\right)^{\prime}+C_{3}(x) Y_{0}\left(\beta r_{2}\right)\right) \\
& \beta^{2}=\frac{\rho \omega^{2} \operatorname{Re}\left(1+i \omega \alpha_{1}\right)}{\Gamma\left(1+i \omega \alpha_{2}\right)}
\end{aligned}
$$


where $Y_{n}$ is an $n$-order Bessel function of the second kind. Substituting (3.3) in the second relation (2.2), we obtain an equation for $u_{r}^{*}(r, x)$ whose solution satisfying (2.11) has the form:

$$
\begin{aligned}
u_{r}^{*}(r, x) & =\frac{C_{5}(x)}{r}-\frac{Y_{1}(\beta r)}{\beta} C_{3}^{\prime}(x)-\frac{J_{1}(\beta r)}{\beta} C_{4}^{\prime}(x)-\frac{r}{2 \rho \omega^{2}}\left(p_{s}^{*}\right)^{\prime \prime} \\
C_{5}(x) & =r_{2}\left(\frac{Y_{1}\left(\beta r_{2}\right)}{\beta} C_{3}^{\prime}(x)+\frac{J_{1}\left(\beta r_{2}\right)}{\beta} C_{4}^{\prime}(x)+\frac{r_{2}}{2 \rho \omega^{2}}\left(p_{s}^{*}\right)^{\prime \prime}\right)
\end{aligned}
$$

From conditions (2.7) and (2.8) we have

$$
\begin{gathered}
p_{s}^{*}(x)=p^{*}(x), \quad C_{3}(x)=A_{3}(x)\left(p^{*}\right)^{\prime}, \quad A_{3}(x)=\left[\frac{Y_{1}(\beta a)}{J_{1}(\beta a)}-\frac{Y_{0}\left(\beta r_{2}\right)}{J_{0}\left(\beta r_{2}\right)}\right]^{-1} \frac{1}{\rho \omega^{2} J_{0}\left(\beta r_{2}\right)} \\
C_{1}(x)=A_{1}(x)\left(p^{*}\right)^{\prime}, \quad A_{1}(x)=i \omega J_{0}^{-1}(\gamma a)\left[A_{3} Y_{0}(\beta a)+A_{4} J_{0}(\beta a)+\frac{1-\rho}{\rho \omega^{2}}\right]
\end{gathered}
$$

For $p^{*}(x)$, from (2.7) we obtain the differential equation

$$
\begin{aligned}
& N_{1}(x)\left(p^{*}\right)^{\prime \prime}+N_{2}(x)\left(p^{*}\right)^{\prime}=0 \\
& N_{1}(x)=\frac{J_{1}(\gamma a)}{\gamma} A_{1}-\frac{i \omega}{\beta}\left(Y_{1}(\beta a) A_{3}+J_{1}(\beta a) A_{4}\right)+\frac{i a(\rho-1)}{2 \rho \omega}-\frac{i \omega}{a} A_{5} \\
& N_{2}(x)=\frac{J_{1}(\gamma a)}{\gamma} A_{1}^{\prime}-\frac{i \omega}{\beta}\left(Y_{1}(\beta a) A_{3}^{\prime}+J_{1}(\beta a) A_{4}^{\prime}\right)+\frac{i \omega}{a} A_{6} \\
& A_{5}(x)=\frac{r_{2}}{\beta}\left(Y_{1}\left(\beta r_{2}\right) A_{3}+J_{1}\left(\beta r_{2}\right) A_{4}\right)+\frac{r_{2}^{2}}{2 \rho \omega^{2}}, \quad A_{6}(x)=\frac{r_{2}}{\beta}\left(Y_{1}\left(\beta r_{2}\right) A_{3}^{\prime}+J_{1}\left(\beta r_{2}\right) A_{4}^{\prime}\right)
\end{aligned}
$$

The solution of this equation has the form:

$$
p^{*}(x)=p_{0}+C_{6} \int_{0}^{x} F(x) d x, \quad F(x)=\exp \left(\int \frac{N_{2}(x)}{N_{1}(x)} d x\right)
$$

The integration constant $C_{6}$ can be found from the second condition (2.10):

$$
C_{6}=\left(y_{t} \int_{0}^{1} F(x) d x-\frac{2 i a(1) F(1)}{\omega}\right)^{-1}\left(\frac{2 a(1)}{\gamma} J_{1}(\gamma a(1)) C_{1}(1)-p_{0} y_{t}\right)
$$

Relations (3.1)-(3.5) make it possible to calculate the flow characteristics most important for applications, including blood flow parameters important for diagnostics: the volume flow-rate $Q(t, x)$, the wall shear stress $\tau_{w}(t, x)$, the linear velocity on the tube axis $v_{x}(t, x)$, and the admittance $Y_{\text {in }}=Q^{*} / p^{*}(x=0)$, where $Q^{*}$ is the flow-rate amplitude. We do not reproduce the final expressions for these values as they are too cumbersome.

In solving the first-approximation problem (2.11)-(2.22), the sequence of operations is similar to that for problem (2.2)-(2.10); therefore, for greater concision, the final cumbersome expressions for the quantities

$$
g(t, r, x)=g^{* *}(r, x) e^{i \omega t}, \quad g=\left\{u_{r}^{(1)}, u_{x}^{(1)}, v_{r}^{(1)} v_{x}^{(1)}, p^{(1)}, p_{s}^{(1)}\right\}
$$

have not been reproduced. 


\section{RESULTS OF NUMERICAL CALCULATIONS AND DISCUSSION}

In the case of a tube with a constant cross-section ( $a=$ const), expressions (3.1)-(3.5) and the similar first-approximation relations can be considerably simplified. In this case, $p^{*}=p_{s}^{*}=$ const and $v_{r}^{*}=u_{r}^{*}=0$. If $a=a(x)$, the distributions of the fluid pressure and velocity field and of the tube wall displacements can be found by integration and are fairly cumbersome functions of the coordinates even in the zeroth approximation. We numerically investigated the effect of the model parameters on the problem solution, in particular, on the integral characteristics $Q(t, x)$ and $Y_{i n}$, the oscillations of the inner diameter of the tube $d=d(t, x)$, and the wall shear stresses $\tau_{w}(t, x)$. When modeling the terminal element, for convenience of physical interpretation, we introduced a wave reflection coefficient $\xi=\xi_{1}+i \xi_{2}$ linked with the dimensionless element admittance by the relation $y_{t}=(1-\xi) /(1+\xi)$ [6]. The value $\xi=1$ corresponds to a closed tube end and $\xi=0$ to the absence of a reflected wave (if at the tube end the conductivities are coordinated).

The ranges of variation of the dimensionless parameters were taken in accordance with the data on the normal human arterial system: $p_{0}=10^{4} \mathrm{~Pa}, \mathrm{Re}=0.1-100, \varepsilon=10^{-2}-10^{-1}$, and $G=10^{5}-10^{6} \mathrm{~Pa}$. When investigating the dependence of the flow characteristics on one of the parameters, the following values of the other parameters were used: $h=H / r_{0}=0.2, \rho=1, \alpha_{1}=\alpha_{2}=0.1, \Gamma=10, \operatorname{Re}=10$, and $\xi=0.5$. The base frequency of the wave $f=\omega /(2 \pi)$ was taken in accordance with the heart contraction frequency $(f=1-1.25 \mathrm{~Hz})$. For all quantities, the dependences shown in the graphs reproduced below were obtained by calculating the corresponding zeroth- and first-approximation values; thus, $f=f^{*}+\varepsilon f^{* *}$. The quantities $f$ were written in the form $f=f^{+} e^{i \varphi}$ and the graphs show the amplitudes $f^{+}$of the corresponding quantities. For a tube of constant cross-section, the error of the calculations in the zeroth approximation did not exceed $1 \%$. Among tubes with a variable cross-section, we considered those determined by the relations

$$
\begin{gathered}
a(x)=a_{2}-\left(a_{1}-a_{2}\right)(x-1), \quad a(x)=\sin \left(k_{1} x+k_{2}\right) \\
k_{2}=\arcsin \left(a_{1}\right), \quad k_{1}=\arcsin \left(a_{2}\right)-k_{2}
\end{gathered}
$$

where $a_{1,2}$ is the dimensionless radius of the tube at $x=0,1$ and $\left(a_{1}-a_{2}\right) / a_{1} \leq 0.01$, which corresponds to normal physiological variations of artery shapes. In these cases, the error in the flow parameters calculated in the zeroth approximation was equal to $2-7 \%$.

Previously, in the literature, regimes without a reflected wave (coordinated conductivities or an infinitely long tube) were investigated [8-11]. The presence of reflection leads to a reverse fluid flow and changes the velocity profiles in both the fluid and the wall. The calculated velocity fields in the tube wall and in the fluid are presented in Fig. 2. The calculations showed that variations of the reflection coefficient within the range $\xi_{1} \in[0 ; 0.25]$ produce only slight changes in the velocity profile. The variations of the fluid volume flow-rate then depend on the Reynolds number and for moderate $\operatorname{Re} \leq 50$ do not exceed $20 \%$ if $\xi_{1} \leq 0.25$. This is related with the fact that reflection leads to a change in the pressure amplitude in the tube as a result of the superposition of the incident and reflected waves, which produces an increase in the tube lumen.

In the literature devoted to the calculation of wave reflection coefficients at artery bifurcations, the problem of conductivity coordination at the bifurcations of at least the main large arteries of the human vascular system has long been discussed [20]. Measurements on specimens [12] show that the conductivities are often uncoordinated, which contradicts ideas about the optimal organization of arterial beds as systems of long-range fluid transport [21]. The calculations performed show that moderate wave reflection in arterial junction regions does not lead to significant deceleration of the fluid flow, which may in part explain the observed non-coordination of the conductivities of the arteries at their branchings.

The wall material stiffness influences the velocity distribution in the fluid: it leads to a certain flattening of the profile at small values of the shear modulus $\Gamma$ and has a considerable effect on the wall displacements (Fig. 3). The volume flow-rate $Q$ remains almost constant due to flow acceleration near the compliant wall and deceleration near the tube axis. The parameters $\alpha_{1}$ and $\alpha_{2}$ affect the fluid flow only slightly. Certain combinations of the wall parameters $\Gamma, \alpha_{1}$, and $\alpha_{2}$ and the relative thickness of the wall have a more pronounced effect on the flow characteristics. In Fig. 4, the dependence $Q(h)$ is presented for various 


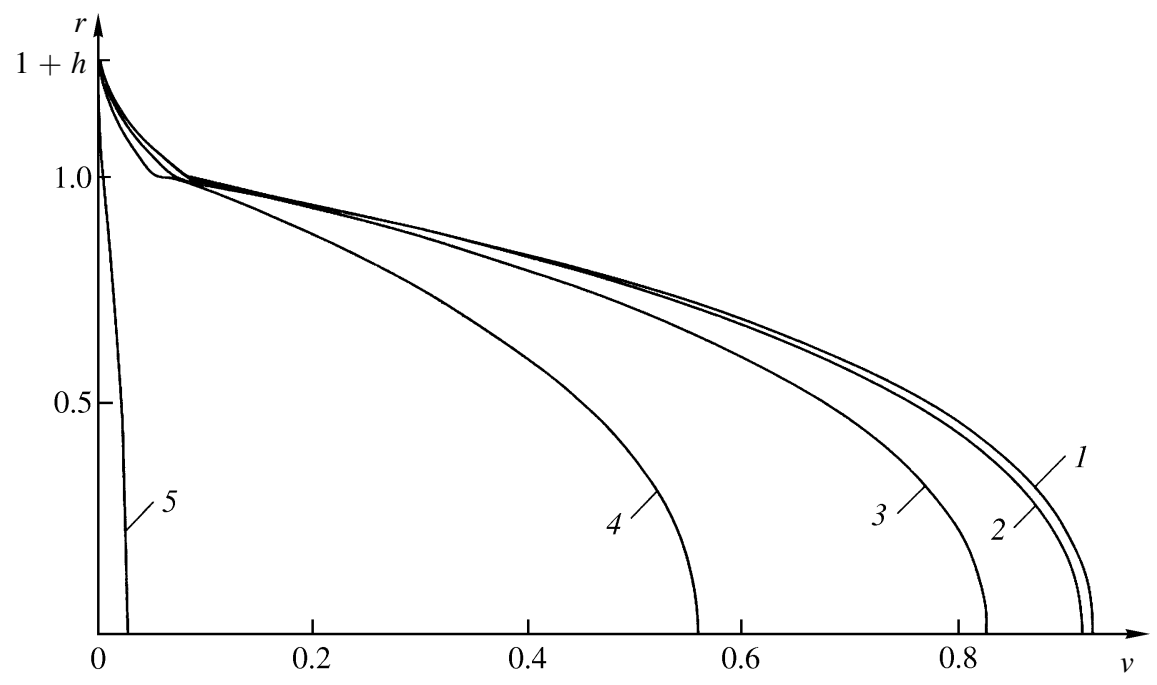

Fig. 2. Profiles of the velocities of the fluid $v_{x}(r)$ and of the wall $u_{x}(r)$ in a tube of a constant cross-section: $\xi=0,0.2,0.4$, $0.6,0.8$ (curves $1-5$ )

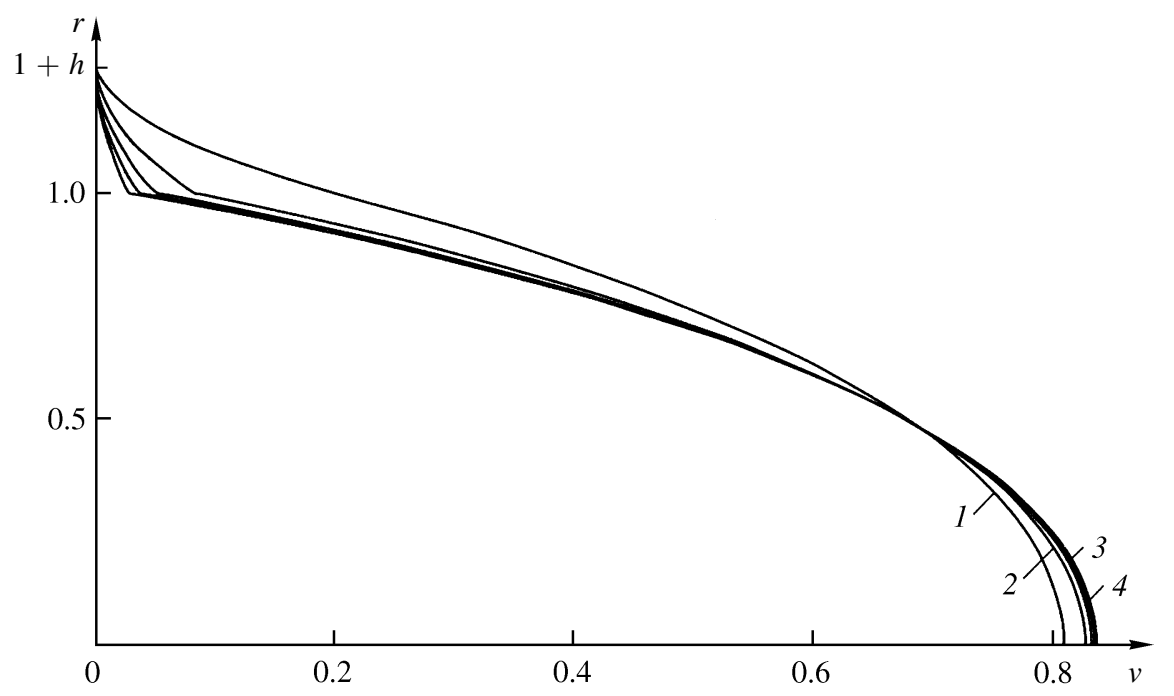

Fig. 3. Profiles of the velocities of the fluid $v_{x}(r)$ and of the wall $u_{x}(r)$ for the values of the dimensionless wall material shear modulus $\Gamma=5,10,20,30$ (curves $1-4$ )

$\Gamma$ values. With increase in the wall thickness and compliance the flow-rate decreases and at $\Gamma, \alpha_{1}$ and $\alpha_{2}$ values limiting for the arterial wall may fall by $15-20 \%$. With increase in the frequency of the incident wave $p_{0}(t)$ the effect becomes less pronounced. For a sufficiently thick wall $(h=0.3-0.5)$, variations of $\alpha_{1}$, $\alpha_{2} \in[0 ; 1]$ produce flow-rate variations in both directions, which do not exceed 5\%. The flow-rate increases with decrease in $\alpha_{2}$ and increase in $\alpha_{1}$. With increase in the Reynolds number the wall shear stress increases monotonically, the $\tau_{w}$ values being higher for a stiffer wall, especially at $\operatorname{Re}>20$ (Fig. 5).

The flow-rate variations related with the wall parameters can be attributed to the interaction of the wall with the fluid flow. In the fluid-tube system part of the kinetic energy of the moving fluid is spent on wall deformation and dissipation. Thus, with increase in the energy transmitted from the flow to the wall, leading to its deformation, the flow-rate decreases. This takes place with increase in the wall compliance (decrease in $\Gamma$ ) and with increase in the relaxation time and decrease in the retardation time of the material. Similar regularities were also detected in collapsed tube flows, where the instability develops due to energy exchange in the fluid-wall system [7, 16]. From (3.3), (3.4) and similar expressions for $u_{r}^{* *}(r, x)$ and $u_{x}^{* *}(r, x)$ there 


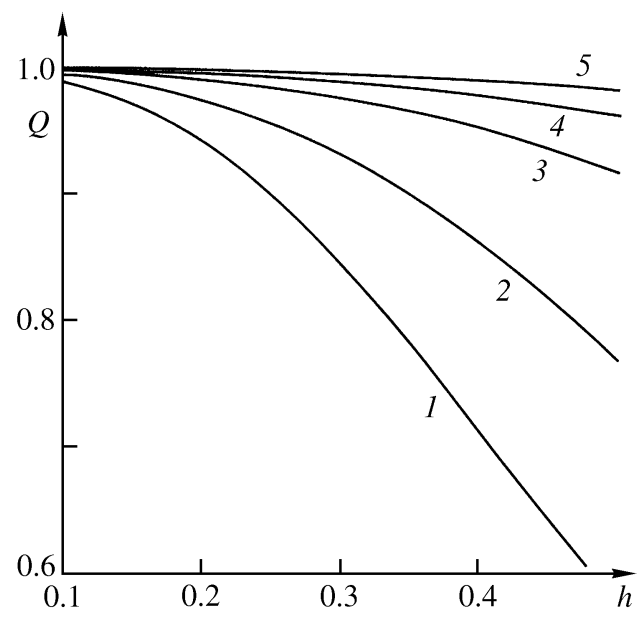

Fig. 4. Dependence of the volume flow-rate on the tube wall thickness $Q(h)$ for $\Gamma=5,10,15,20,25$ (curves $1-5)$

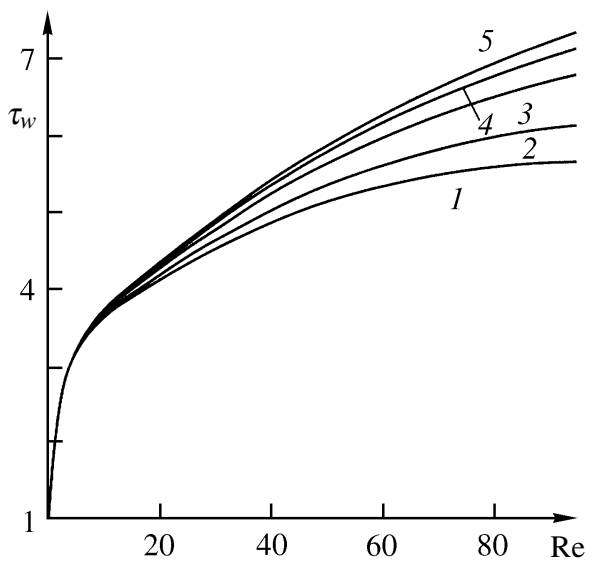

Fig. 5. Dependence of the wall shear stress $\tau_{w}$ on the Reynolds number $\tau_{w}(\operatorname{Re})$ for $\Gamma=5,10,15,20,25$ (curves $1-5$ )

follows the dependence

$$
u_{r, x} \sim \frac{\operatorname{Re}}{\beta^{2} \Gamma}=\frac{1+i \omega \alpha_{2}}{\rho \omega^{2}\left(1+i \omega \alpha_{1}\right)}
$$

On the physiological parameter range the flow-rate variations due to interaction on the fluid-wall interface are moderate. During the development of pathologies associated with thickening of the wall and a simultaneous decrease in its density due to disintegration and the accumulation of fatty components, as the calculations show, the decrease in the blood flow-rate is comparable with the decrease in $Q$ due to a decrease in the admittance $y_{t}$ at $\xi_{1}=0.2-0.3$. Normally, the blood volume flow-rate may be expected to be restored due to active arterial lumen regulation, including feedback controlled by the parameters of the reflected wave [22]; therefore, the functions $Q\left(\Gamma, \alpha_{1}, \alpha_{2}\right)$ investigated are important for blood flow both in diseased vessels and in the tubes of technical devices. Moreover, dependence (4.1) is of interest in connection with a recently proposed method of calculating the intravascular pressure from a noninvasive ultrasound recording of the radial displacements of the vascular wall $d(t)=a\left(x_{0}\right)+2 u_{r}\left(t, a\left(x_{0}\right), x_{0}\right)$ in an arbitrary cross-section $x_{0}$ during the passage of a pulse wave. The measurement results presented in [23] show good agreement between the maximum (systolic), minimum (diastolic) and mean pressures measured directly in one radial artery of a patient (by introducing a catheter with a micromanometer into the artery) and those calculated for the other radial artery from the diameter oscillations $d(t)$. The dynamics of the $p(t)$ and $d(t)$ curves were 


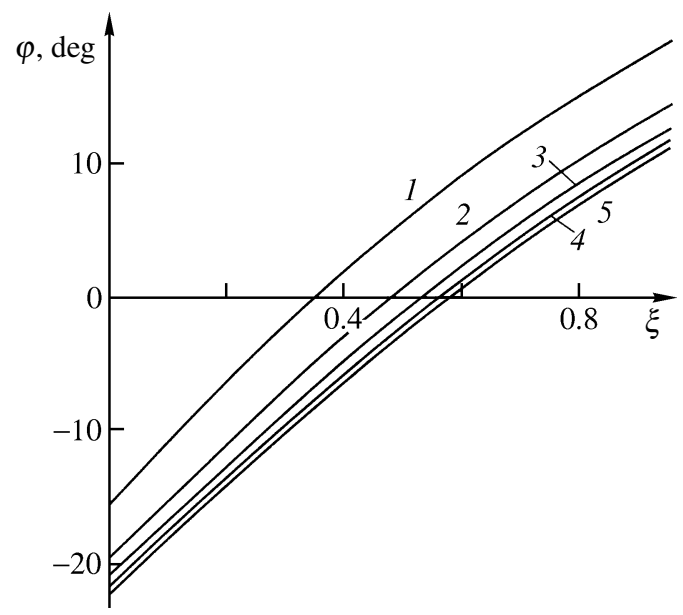

Fig. 6. Dependence of the volume flow-rate phase $\varphi$ on the pressure wave reflection coefficient $\xi_{1}$ for $\Gamma=5,10,15,20,25$ (curves 1-5)

also similar. The error of the noninvasive method is equal to $\pm 15-20 \%$ and increases up to $\pm 25 \%$ at high mean pressures $(>100 \mathrm{~mm} \mathrm{Hg})$.

Thus, for vascular wall pathologies associated with increased arterial pressure, the method of calculating the $p(t)$ curves from records of the $d(t)$ curves should be made more precise, for example, by performing calculations on the basis of the above-proposed model with the dependence $p_{0}(t)$ typical of arteries and values of the parameters $\Gamma, \alpha_{1,2}$, and $\xi$ characteristic of various pathological conditions.

For medical diagnostic purposes, as an important and informative parameter, the volume flow-rate phase $\varphi$ can be used. Variations of the wall parameters affect the phase only slightly $\left(\delta \varphi \sim 4-8^{\circ}\right)$, whereas a variation of the reflection coefficient $\xi$ leads to variations $\delta \varphi \sim 40-50^{\circ}$ and to a change in the sign of the quantity $\varphi$. Thus, depending on the wave reflection conditions, the flow-rate oscillations may either lag behind or lead the pressure oscillations in phase. A similar dependence is known, and has been more than once investigated, for the aorta whose bifurcation has a reflection coefficient $\xi_{1}=0.25-0.35$, which is high for the arterial system [12]. The function $\varphi(\xi)$ increases in all the cases studied (Fig. 6), but its position and the quantity $\varphi(0)-\varphi(1)$ depend on the wave frequency. Since the pulse wave in arteries can be represented as the sum of the first few Fourier harmonics, it is reasonable to investigate the phase spectrum of the volume flow-rate. From calculations based on the above-obtained relations we can find the characteristics $\varphi(\omega)$ for various reflection conditions $\xi_{1} \in[0 ; 1]$. The preliminary results presented in Fig. 6 show that analysis of the phase spectrum can be used for diagnosing pathologically high reflection coefficients, even without previously investigating the mechanical properties of the wall of the artery studied, and using only the $p(t)$ and $Q(t)$ curves synchronously recorded for this vessel by available noninvasive methods.

The $d(t)$ curves recorded by ultrasound methods and describing the wall oscillations are used for calculating the intravascular pressure $p(t)$ curves and the subsequent analysis of the pulse waves; therefore, for estimating the diagnostically significant information it is important to investigate the effect of the wall material parameters and the conditions of flow and wave reflection. The results of our analysis show that the present methods of calculating the $p(t)$ curves from the measured values of $d(t)$ [23] should be refined, at least in the case of an increased mean arterial pressure and accompanying vascular pathologies.

Summary. The propagation of waves in a thick-walled tube of incompressible isotropic viscoelastic material, filled with a viscous incompressible fluid, is investigated with reference to pulse wave propagation in arteries. At the tube end there is an element that models the downstream vascular bed and determines the wave reflection conditions. The problem solution is obtained in the form of expansions in a small parameter equal to the ratio of the radial displacement of the wall to the inner radius of the tube. Relations for calculating the linear velocity and volume flow-rate of the fluid, the tube wall displacement, the wall shear stress, and the spectrum of the inlet admittance of the tube-terminal element system are obtained. It is 
shown that certain pulse wave parameters, such as the phase spectrum of the blood volume flow-rate $Q(t)$, depend only weakly on the individual parameters of the vascular wall and strongly on the wave reflection conditions. Therefore, these parameters may be proposed for diagnosing intraorganic blood circulation conditions (quantities $y_{t}$ ) from the results of ultrasound measurements of $Q(t)$ and the wall oscillations $d(t)$ in the artery feeding the organ.

\section{REFERENCES}

1. M. F. O'Rourke, R. P. Kelly, and A. P. Avolio, The Arterial Pulse, Lea and Febiger, Philadelphia (1992).

2. I. N. Moiseeva and S. A. Regirer, "Some properties of pulse wave reflection in arteries," Fluid Dynamics, 28, No. 4, 536-540 (1993).

3. Y. Y. Wang, S. L. Chang, Y.E. Wu, T. L. Hsu, and W. K. Wang, "Resonance. The missing phenomenon in hemodynamics," Circ. Res., 69, No. 1, 246-249 (1991).

4. N. N. Kizilova, "Reflection of pulse waves and resonance characteristics of arterial beds," Fluid Dynamics, 38, No. 5, 772-781 (2003).

5. N. N. Kizilova, "Investigation of the pressure-flow-rate dependence and the parameters of incident and reflected pressure waves in arterial beds," Akust. Vestnik, 7, No. 1, 50-61 (2004).

6. W. R. Milnor, Hemodynamics, Williams and Wilkins, Baltimore, etc. (1989).

7. M. Hamadiche and N. N. Kizilova, "Temporal and spatial instabilities of the flow in the blood vessels as multilayered compliant tubes," Intern. J. Dynam. Fluids, 1, No. 1, 1-23 (2005).

8. J.R. Womersley, "An elastic tube theory of pulse transmission and oscillatory flow in mammalian arteries," Tech.Report TR-56-614 (1957).

9. M. G. Taylor, "The input impedance of an assembly of randomly branching elastic tubes," Biophys. J., 6, No. 1, 29-51 (1966).

10. I. Mirsky, "Wave propagation in a viscous fluid contained in an orthotropic elastic tube," Biophys. J, 7, No. 2, 165-186 (1967).

11. H. B. Atabek, "Wave propagation through a viscous fluid contained in a tethered, initially stressed, orthotropic elastic tube," Biophys. J, 8, No. 5, 626-649 (1968).

12. A. P. Avolio, "Multi-branched model of the human arterial system," Med. and Biol. Eng. and Comput., 18, No. 6, 709-718 (1980).

13. V. A. Levtov, S. A. Regirer, and N. Kh. Shadrina, Blood Rheology [in Russian], Meditsina, Moscow (1982).

14. R. H. Cox, "Comparison of linearized wave propagation models for arterial blood flow analysis," J. Biomech., 2, No. 3, 251-265 (1969).

15. A. W. Khir and K. H. Parker, "Measurements of wave speed and reflected waves in elastic tubes and bifurcations," J. Biomech., 35, No. 6, 775-783 (2002).

16. S. Naili and C. Ribreau, "Wall shear stress in collapsed tubes," Eur. Phys. J, 5, No. 1, 95-100 (1999).

17. H. Alderson and M. Zamir, "Smaller, stiffer coronary bypass can moderate or reverse the adverse effects of wave reflections," J. Biomech., 34, No. 11, 1455-1462 (2001).

18. M. F. O'Rourke, A. Pauca, and X.-J. Jiang, "Pulse wave analysis," Brit. J. Clin. Pharmacol., 51, No. 6, 507-522 (2001).

19. V. Shankar and V. Kumaran, "Asymptotic analysis of wall modes in a flexible tube revisited," Eur. Phys. J, Ser.B, 19, No. 4, 607-622 (2001).

20. J. J. Wang and K. H. Parker, "Wave propagation in a model of the arterial circulation," J. Biomech., 37, No. 4, $457-470$ (2004).

21. C. M. Quick, D. A. O'Hara, and A. Noordergraaf, "Pulse wave reflection and arterial inefficiency," Proc. 17th IEEE Annual Conf. "Engineering in Medicine and Biology", 1, 97-98 (1995).

22. C. M. Quick, D. S. Berger, and A. Noordergraaf, "Arterial pulse wave reflection as feedback," IEEE Trans. BME, 49, No. 5, 440-445 (2002).

23. K. Belani, M. Ozaki, J. Hynson, et al., "A new noninvasive method to measure blood pressure," Anesthesiology, 91, No. 3, 686-692 (1999).

E-mail: nnk_@bk.ru 